Volume 9, No.1.4, 2020

International Journal of Advanced Trends in Computer Science and Engineering

Available Online at http://www.warse.org/IJATCSE/static/pdf/file/ijatcse7991.42020.pdf

https://doi.org/10.30534/ijatcse/2020/7991.42020

\title{
A Review on Brain Glioma Segmentation in MRI images using Deep Learning
}

\author{
Nurul Nadhirah Md Nasriy ${ }^{1}$, Siti Salasiah Mokri ${ }^{2}$, Ashrani Aizuddin Abd Rahni ${ }^{3}$ \\ ${ }^{1}$ Department of Electrical, Electronic and Systems Engineering, Faculty of Engineering and Built Environment, \\ Universiti Kebangsaan Malaysia, 43600 UKM Bangi, Selangor, Malaysia, p102811@ siswa.ukm.edu.my \\ ${ }^{2}$ Department of Electrical, Electronic and Systems Engineering, Faculty of Engineering and Built Environment, \\ Universiti Kebangsaan Malaysia, 43600 UKM Bangi, Selangor, Malaysia, siti1950@ukm.edu.my \\ ${ }^{3}$ Department of Electrical, Electronic and Systems Engineering, Faculty of Engineering and Built Environment, \\ Universiti Kebangsaan Malaysia, 43600 UKM Bangi, Selangor, Malaysia, ashrani@ukm.edu.my
}

\begin{abstract}
Gliomas are a kind of tumor that starts in the neuroglia cells of the brain or the spine. Gliomas constitute about 80 percent of all malignant brain tumors and 30 percent of all brain and central nervous system tumors. An accurate segmentation of gliomas is crucial in order to diagnosis brain tumour as well as to perform the proper treatment planning and management. Gliomas segmentation involves determination of their locations and sizes in the brain. Brain glioma segmentation in Magnetic resonance imaging (MRI) images is important to assist diagnosis, treatment planning and surgical navigation. However, manual segmentation is time consuming and is prone to bias. Thus, robust software based automatic segmentation methods are required. Machine learning based techniques particularly deep learning methods have been proposed for brain glioma segmentation and they are successful. This paper presents a review on segmentation of brain glioma in MRI images using various recent and state-of-the-art deep learning methods. These methods are identified and their performances are compared.
\end{abstract}

Key words: Brain tumor, Glioma, Segmentation, Magnetic Resonance Imaging, Deep Learning.

\section{INTRODUCTION}

Gliomas have the highest mortality rate in common brain tumors [1]. These neoplasms can be divided into Gliomas of High Grade (HGG) and Gliomas of Low Grade (LGG), where the former is more violent than the latter [1][2]. However, after the first diagnosis many patients will not last longer than 14 months on average under assisted treatments and medications. Existing therapies for brain tumors include surgery, radiation, radiotherapy or a combination of these treatments [3], [4]. In clinical practice, magnetic resonance imaging (MRI) is especially useful for determining gliomas, since it is possible to obtain MRI sequences that provide additional information. MRI is a comprehensively used form of imaging for observing these tumors. The enormous proportion of data produced by MRI imaging facilitates manual segmentation and the implementation of precise quantitative estimates in clinical practice. In addition, MRI is especially helpful in clinical practice in evaluating gliomas [4]. Example images of LGG and HGG Gliomas are as shown in Figure 1 and 2.

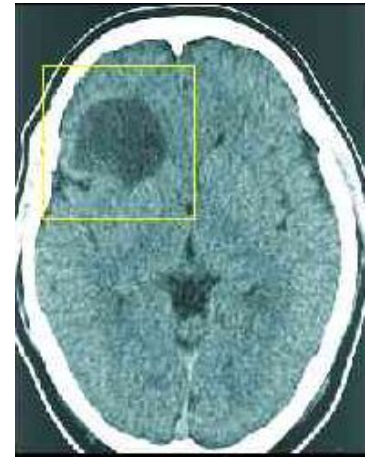

Figure 1: LGG [17]

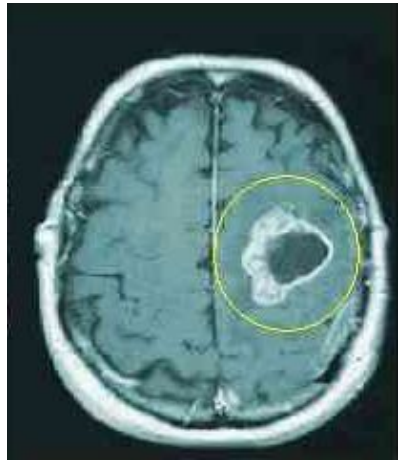

Figure 2: HGG [17]
Precise segmentation of gliomas and their structures is not only critical for care planning but also for follow-up evaluation. Manual segmentation, however, is tedious and vulnerable to inter- and intra-rater errors which are hard to characterize [5], [6]. However, most doctors typically use rough measures for the assessment process. Because of these reasons, exact semi-automatic or automated methods are needed [7].

Gliomas segmentation in MRI images is, however, a difficult and challenging task, as the location, structure and shape of these tumors is highly variable [8]. The tumor mass also influences the structure of the normal tissues that surround it. Additionally, MRI images may pose some problems, such as in-homogeneity intensity [9], or specific ranges among the same sequences and acquisition scanners [10].

Deep learning related approaches have become effective tools for automatically learning features in various medical 
imaging applications including segmentation of brain tumors, segmentation of brain tissues, segmentation of lung tumors and segmentation of the liver. The task of segmentation of brain glioma is a difficult problem, as the location, structure and shape of gliomas differ among individual patients. This poses a challenge in creating an effective model using deep learning for segmentation of gliomas.

This paper presents the review of previous methods for brain tumor segmentation utilizing deep learning methods. The rest of the paper is organized as follows: Section 2 describes the proposed methods by the previous researchers. Section 3 shows comparison of these methods and their performance. Lastly, Section 4 presents the conclusions.

\section{LITERATURE REVIEW FOR METHODS}

Brain tumor segmentation using deep learning is a fully automated, objective and reproducible method. Brain tumor segmentation especially of gliomas presents many challenges in designing an accurate deep learning segmentation model due to several factors such as variability in terms of the size, shape, regularity, location as well as the heterogeneous appearance of these brain tumors. According to previous literature, among the deep learning methods proposed for brain tumor segmentation are the basic convolutional neural network, the hierarchical framework, the cascade approach, the trunk network and the pyramid pool (WRN-PPNet) network.

\subsection{Basic Convolutional Neural Network Method}

Convolutionary Neural Networks (CNNs) are an alternative type of neural network, which can be used to reduce spectral variations and model spectral correlations in signals [11]. The approach is based on deep neural networks (DNN) and learns different features for the segmentation of brain tumors [12][13]. Yi-Ju Chang et al. [14] introduced a method to provide automatic brain tumor segmentation using a $2 \mathrm{D}$ convolutional neural network in 2016. The training data sets were obtained from the BraTs 2018 challenge [14]. Four types of images were used in the network which are T1, T1ce, T2 and FLAIR images, as input images. The segmentation network used was the SegNet. In order to boost the variability of the images, the angles, contrast, and brightness of the training images were randomly changed. They have shown that the augmentation of enhancing variability of the images has better outcomes than images without augmentation. Evaluation on the testing data is only possible with the online evaluation tool. Results are presented by the tool mainly in the form of well-known Dice Score [15]. During the validation phase, an optimized segmentation method was applied to the validation datasets. They achieved an average dice coefficients are $0.766,0.896,0.824$ for the three subsets, the enhancing tumor, the tumor core, and the whole tumor, respectively. The proposed deep learning models are effective for the segmentation of brain tumors and allow to obtain high accurate results. Moreover, the proposed models could help the physician experts to reduce the time of diagnostic [16]. Neural Network accuracy also relies on the right choice of $\mathrm{f}$ eatures and proper combination of different layers [17],[18].

Saqib Qamar et al. [19] proposed a method where numerous densely connected blocks were designed to exploit the advantages of $\mathrm{CNN}$ to boost the performance of the segmentation model in non-enhancing tumor (NET), enhancing tumor (ET), and peritumoral edema (PE). This method was introduced as 3D hyper-dense Convolutional Neural Network (CNN) in 2018. This architecture adopts a 3D Fully Convolutional Network (FCN) architecture that is used for volumetric prediction from end to end. Their findings were very similar to the facts reported on the field. This approach is preferable to present 3D-based approaches for the segmentation of MRI brain tumors when it comes to compactness, time and parameter efficiency. Throughout this study, the capacity of densely connected blocks throughout 3D segmentation tasks was explored by considering multiscale receptive fields for the precise classification of voxels. The experimental findings revealed the advantages of the proposed design over state-of-the-art models without advanced tricks. Therefore, they concluded that the proposed model has great potential for segmentation of MRIs or other tasks for segmentation of medical images. They received 0.84 , 0.81 and 0.87 Dice ratings for the tumor center, strengthening tumor segmentation and total tumor segmentation, respectively.

In addition to endorsing the vector machine classifier approach for brain tumor segmentation, an architecture for convolutional neural network-based learning transfer was built in 2019 by Bin Cui et al. [20]. The algorithm is composed of two cascaded steps. The first stage is the training process for the $\mathrm{CNN}$ to learn and mark a mapping from the images in the data sets. Therefore, during the testing process, the projected CNN label output is used for a more accurate segmentation before being submitted to an SVM classifier. In contrast, they have shown that this method is better than separate segmentation based on $\mathrm{CNN}$, or separate segmentation based on SVM. The structure of the proposed architecture consists of two main phases; the first is pre-processing, extraction of functionality, training of CNN and SVM while the second is testing and producing the effects of segmentation. Compared with other methods the proposed method has a reasonable efficiency. This also greatly outperformed the pure application of CNN or SVM to identify tumors. They have obtained a dice score of 0.88 for the value of Dice Coefficient of Similarity (DCS), 0.83 for Positive Predictive Value (PVV), and the sensitivity is about 0.89 . Table 1 shows the performance of this method as compared to CNN or SVM alone. The results show that the combination of CNN and SVM (CNN-SVM) achieves the best segmentation accuracy. 
Table 1: Segmentation result comparison

\begin{tabular}{|l|c|c|c|}
\hline \multicolumn{1}{|c|}{ Method } & CNN-SVM & CNN & SVM \\
\hline DSC & $0.88 \pm 0.04$ & $0.79 \pm 0.09$ & $0.70 \pm 0.11$ \\
\hline PPV & $0.83 \pm 0.04$ & $0.79 \pm 0.10$ & $0.78 \pm 0.06$ \\
\hline Sensitivity & $0.89 \pm 0.02$ & $0.81 \pm 0.11$ & $0.83 \pm 0.12$ \\
\hline
\end{tabular}

\subsection{Hierarchical framework method}

Previous research has shown that it is possible to resolve difficulties in studying highly nonlinear and complex patterns in data by applying a deep or hierarchical architecture. Zhe Xiao et al. [21] has suggested a novel approach for the segmentation of brain tumors based on a hierarchical structure. This consists of classification based on the pre-processing, deep learning network, as well as post-processing stages. The pre-processing stage is used to extract image patches for each MRI image, and the gray-level image patch sequences are collected to make it the input to the deep learning network. To extract the high-level abstract function from the data, the deep learning network-based classification stage is implemented through a stacked auto encoder network and the extracted function is used to classify image patches. After mapping the result of the classification to a binary image, the post-processing stage consists of a morphological filter to obtain the final result of the segmentation. In order to evaluate the proposed architecture, the approach was applied to segment the brain tumor for the actual ground reality of the case. Their tests have shown that the division technique suggested for brain tumor segmentation is effective. The proposed technique will achieve higher classification accuracy and gets a good matching rate between the product of segmentation and the ground reality. The average accuracy of $96.24 \%$ between the effects of this proposed method and the ground reality.

S'ergio Pereira et al. [22] conducted a study that uses a completely convolutional network with a hierarchical approach for brain tumor segmentation. The evaluation is conducted on the 2013 data set for the Brain Tumor Segmentation Challenge, and the Dice Score Coefficient, Positive Predictive Value, and Sensitivity metrics were reported. Their findings showed the advantages of first segmenting the entire tumor, over all tissues in one stage. In fact, the tumor center also benefited from such an approach that was explained by the high data disparity found between the tumor and normal tissues, which is mitigated by taking the whole tumor into account. A hierarchical approach for the segmentation of brain tumors using Fully Convolutional Neural Networks (FCN) was proposed in this paper. Such networks allow a complete patch to be segmented, thus achieving better regularization. This approach has been found to be helpful for the segmentation of brain tumors using FCN, not only to detect the whole tumor, but also to better delineate the core and enhancing tumor region. In terms of the DSC of the complete and enhancing tumor regions, as well as the core sensitivity and enhancing tumor regions, the improvements over the single stage approach were found to be statistically important.

\subsection{Cascade approach method}

A brain tumor segmentation using Cascade approach was introduced by Zahra Sobhaninia et al. [23]. While the use of a two scale method increases the precision of brain tumor segmentation, difficulties in brain tumor segmentation such as tumor position and similarity of brightness level with tissue result in poor outcomes. The cascaded method is suggested to tackle these problems [24].

With multiple scales of images, this Cascade approach is used to obtain both local and global views and to help the network achieve higher precision. Results from the experiment show that using multiple scales and using two cascade networks is advantageous. This architecture is a multi-scale, LinkNet-based network usually used for semanthetic segmentation tasks [25]. LinkNet is an end-to - end encoder decoder architecture that is basically, respectively, a discriminative-generative network. In addition, this paper uses a cascaded model structure based on the LinkNet dual-scale (DSLN) to enhance the segmentation of brain tumors. The score of the test is calculated using (Dice) and standard mean, Intersection over Union (IoU). When the output map of the first DSL Net is concatenated as input for the second DSL Net with the input image, the output results are better accurate and closer to ground-truth. In Dice and Mean IOU, respectively, the results obtained $78 \%$ and $88 \%$ by training a Dual-Scale LinkNet. Such measurements are performed after the second network has been equipped with the first network output concatenated with the input image.

\subsection{WRN PPNET}

Yu Wang et al [26] has suggested an automated system, called the Wide Residual and Pyramid Pool Network (WRN-PPNet), which can segment gliomas automatically. The proposed method is validated on the BRATS2015 databases [27]. Firstly, a small number of two-dimensional (2D) slices are obtained from images of three-dimensional ( 3D) brain tumor MRI. The 2D slices are then standardized and positioned in the WRN-PPNet model, and the model provides the effects of tumor segmentation. Finally, the WRN-PPNet's dice coefficient (Dice), sensitivity coefficient (Sensitivity) and predictive positive value (PPV) coefficient are used to quantitatively evaluate the results. The experimental results show that the proposed approach is simple and stable compared to the other state-of-the-art methods, and the average Dice, Sensitivity and PPV are 0.94, 0.92 and 0.97 respectively on the randomly selected test data. Pre-processing techniques involve standardizing the strength and increasing the data. The WRN module is implemented in the designed method to resolve the reuse of the attenuation function induced by deep ResNet, and the shallow WRN 
module has the output near deep ResNet. The PPNet module retains feature maps of a global context. Instead they are paired with the WRN module function maps. Around the same time, the original inputs are again inserted into the network to improve the representation of features relevant to the efficiency of segmentation of the model. To determine the segmentation effects, three metrics, including dice coefficient, sensitivity coefficient and PPV coefficient are applied [28]. Finally, on the same results, the method proposed is compared with the other state-of-the-art methods and the manually delineated ground reality. The experimental results indicate that the proposed approach can produce more reliable and faster results in segmentation.

\section{REVIEW TABLE}

In this section, the segmentation techniques of brain tumour as proposed by the previous researchers are compiled in Table 2. Accordingly, their performances are compared and evaluated.

\section{CONCLUSIONS}

Gliomas are most commonly diagnosed using magnetic resonance imaging (MRI) images while manual segmentation of the tumours is the common practice to evaluate and quantify the abnormal growth of these tissues. However manual segmentation by the physicians is vulnerable to flaws, time consuming and often produces errors. Due to that reason, automated brain tumor segmentation using deep learning methods have been explored by many researchers and are mostly applicable for diagnosis of this disease. This paper has reviewed various deep learning based methods for brain gliomas segmentation in MRI images and their performances are compared. Automated brain tumor segmentation method eases the laborious work faced by the medical practitioners.

\section{ACKNOWLEDGEMENT}

The authors would like to sincerely thank the Ministry of Higher Education Malaysia and Universiti Kebangsaan Malaysia under Grant University Research GUP-2019-023. 
Nurul Nadhirah Md Nasriy et al., International Journal of Advanced Trends in Computer Science and Engineering, 9(1.4), 2020,569 - 575

Table 2 : Method and performance evaluation of brain tumor segmentation using deep learning

\begin{tabular}{|c|c|c|c|c|}
\hline Author & Year & Paper Tittle & Method & Performance Evaluation \\
\hline $\begin{array}{l}\text { Yi-Ju Chang, } \\
\text { Zheng-Shen } \\
\text { Lin, Tsai-Ling } \\
\text { Yang, Teng-Yi } \\
\text { Huang }\end{array}$ & 2018 & $\begin{array}{l}\text { Automatic } \\
\text { segmentation of } \\
\text { brain tumor from } \\
\text { 3D MR images } \\
\text { using a 2D } \\
\text { convolutional } \\
\text { neural network }\end{array}$ & $\begin{array}{l}\text { 2D convolutional } \\
\text { neural network }\end{array}$ & $\begin{array}{l}\text { The accuracy of the validation } \\
\text { results obtained with the optimized } \\
\text { method was approximately } 0.9 \text { (the } \\
\text { Dice coefficient of WT) but the } \\
\text { segmentation of the enhancing } \\
\text { tumor region was not as accurate } \\
\text { (the Dice coefficient of ET: } 0.766 \text { ). }\end{array}$ \\
\hline $\begin{array}{l}\text { Saqib Qamar } \\
\text { Hai Jin } \\
\text { Ran Zheng } \\
\text { Parvez Ahmad }\end{array}$ & 2018 & $\begin{array}{l}\text { 3D Hyper-dense } \\
\text { Connected } \\
\text { Convolutional } \\
\text { Neural Network } \\
\text { for Brain Tumor } \\
\text { Segmentation }\end{array}$ & $\begin{array}{l}\text { 3D hyper-dense } \\
\text { Convolutional } \\
\text { Neural Network } \\
(\mathrm{CNN})\end{array}$ & $\begin{array}{l}\text { Tumor core dice ratings of } 0.84 \text {, } \\
0.81 \text { and } 0.87 \text {, improving tumor } \\
\text { segmentation and full tumor, } \\
\text { respectively. }\end{array}$ \\
\hline $\begin{array}{l}\text { Bin Cui } \\
\text { Mingchao Xie } \\
\text { Chunxing } \\
\text { Wang }\end{array}$ & 2019 & $\begin{array}{l}\text { A Deep } \\
\text { Convolutional } \\
\text { Neural Network } \\
\text { Learning } \\
\text { Transfer to } \\
\text { SVM-Based } \\
\text { Segmentation } \\
\text { Method for Brain } \\
\text { Tumor }\end{array}$ & $\begin{array}{l}\text { Convolutional } \\
\text { neural network }\end{array}$ & $\begin{array}{l}\text { The DSC value of the approach we } \\
\text { proposed is approximately } 0.88 \\
\text { compared to CNN }(0.79) \text { and } \\
\operatorname{SVM}(0.70) \text { respectively. } \\
\text { Compared with CNN }(0.79) \text { and } \\
\operatorname{SVM}(0.78) \text {, the PPV is about } 0.83 \text {, } \\
\text { and the Sensitivity is about } 0.89 \\
\text { relative to CNN }(0.81) \text { and } \\
\operatorname{SVM}(0.83) \text {. }\end{array}$ \\
\hline $\begin{array}{l}\text { Zhe Xiao, } \\
\text { Ruohan Huang, } \\
\text { Yi Ding, Tian } \\
\text { Lan, } \\
\text { RongFeng } \\
\text { Dong, } \\
\text { Zhiguang Qin } \\
\text { Xinjie Zhang, } \\
\text { Wei Wang }\end{array}$ & 2016 & $\begin{array}{l}\text { A deep learning } \\
\text { based } \\
\text { segmentation } \\
\text { method for } \\
\text { Brain tumor in } \\
\text { MR images }\end{array}$ & $\begin{array}{l}\text { Hierarchical } \\
\text { framework }\end{array}$ & $\begin{array}{l}\text { The average percentage accuracy } \\
\text { between the results of this } \\
\text { proposed system and the ground } \\
\text { truth is } 96.24 \% \text {. }\end{array}$ \\
\hline $\begin{array}{l}\text { Zahra } \\
\text { Sobhaninia, } \\
\text { Safiyeh Rezaei, } \\
\text { Nader Karimi, } \\
\text { Ali Emami, } \\
\text { Shadrokh } \\
\text { Samavi }\end{array}$ & 2020 & $\begin{array}{l}\text { Brain Tumor } \\
\text { Segmentation by } \\
\text { Cascaded Deep } \\
\text { Neural Networks } \\
\text { Using } \\
\text { Multiple Image } \\
\text { Scales }\end{array}$ & $\begin{array}{l}\text { Cascade } \\
\text { approach }\end{array}$ & $\begin{array}{l}\text { Changing the loss function would } \\
\text { have a positive effect on the } \\
\text { efficiency of the network and will } \\
\text { raise the mean Dice score from } \\
73.1 \% \text { to } 76.6 \% \text {. }\end{array}$ \\
\hline $\begin{array}{l}\text { Yu Wang } \\
\text { Changsheng Li } \\
\text { Ting Zhu } \\
\text { Chongchong } \\
\text { Yu }\end{array}$ & 2019 & $\begin{array}{l}\text { A Deep Learning } \\
\text { Algorithm for } \\
\text { Fully Automatic } \\
\text { Brain Tumor } \\
\text { Segmentation }\end{array}$ & $\begin{array}{l}\text { Wide residual \& } \\
\text { pyramid pool } \\
\text { network } \\
\text { (WRN-PPNet) }\end{array}$ & $\begin{array}{l}\text { On the randomly selected test data } \\
\text { the average Dice, Sensitivity and } \\
\text { PPV can reach } 0.94,0.92 \text { and } 0.97 \\
\text { respectively. }\end{array}$ \\
\hline
\end{tabular}




\section{REFERENCES}

1. S. Bauer et al. A survey of MRI-based medical image analysis for brain tumor studies, Physics in Medicine and Biology, Vol. 58, no. 13, pp. 97-129, 2013.

2. D. N. Louis et al. The $\mathbf{2 0 0 7}$ who classification of tumors of the central nervous system, Acta Neuropathological, Vol. 114, no. 2, pp. 97-109, 2007. https://doi.org/10.1007/s00401-007-0243-4

3. E. G. Van Meir et al. Exciting new advances in neuro-oncology: The avenue to a cure for malignant glioma, CA: A Cancer Journal for Clinicians, Vol. 60, no. 3, pp. 166-193, 2010.

4. R. Vinoth and C. Venkatesh. Segmentation and Detection of Tumor in MRI images Using CNN and SVM Classification. In Conference on Emerging Devices and Smart Systems (ICEDSS), 2018, pp. 21-25.

5. S. A. Agnes, and J. Anita. 3D Lung Segmentation on CT images using Region Based Method, International Journal of Advanced Trends in Computer Science and Engineering, pp. 1156-1161, 2019.

https://doi.org/10.30534/ijatcse/2019/24842019

6. R. S. Ahmed, J. Liu, Z. Fei and M. Zahid. Automated segmentation of whole cardiac CT images based on deep learning, International Journal of Advanced Computer Science and Applications, Vol. 9(4), 2018.

7. B. Menze et al. The multimodal brain tumor image segmentation benchmark (BRATS), IEEE Transactions on Medical Imaging, Vol. 34, no. 10, pp. 1993-2024, 2015.

8. D. Sherlin, and D. Murugan. Brain Tumor segmentation using modified Fuzzy metric based Approach with Adaptive Technique, International Journal of Advanced Computer Science and Applications, Vol. 8(6), 2019. https://doi.org/10.30534/ijatcse/2019/08862019

9. N. J. Tustison et al. N4ITK: Improved n3 bias correction, IEEE Transactions on Medical Imaging, Vol. 29, no. 6, pp. 1310-1320, 2010.

10. L. G. Nyúl, J. K. Udupa, and X. Zhang. New variants of a method of MRI scale standardization, IEEE Transactions on Medical Imaging, Vol. 19, no. 2, pp. 143-150, 2000.

11. T. N. Sainath, A. Mohamed, B. Kingsbury and B. Ramabhadran, "Deep convolutional neural networks for LVCSR," 2013 IEEE International Conference on Acoustics, Speech and Signal Processing, 2013, pp. 8614-8618.

12. M. Havaei, A. Davy, D. Warde-Farley, A. Biard, A. Courville, Y. Bengio, ... and $\mathrm{H}$. Larochelle. Brain tumor segmentation with deep neural networks. Medical image analysis, Vol. 35, pp. 18-31, 2017.

13. J. Amin, M. Sharif, M. Yasmin, and S.L. Fernandes. Big data analysis for brain tumor detection: Deep convolutional neural networks. Future Generation Computer Systems, Vol. 87, pp. 290-297, 2018.
14. Y.J. Chang, Z.S. Lin, T.L. Yang, and T.Y. Huang. Automatic segmentation of brain tumor from 3d mr images using a 2d convolutional neural network. In International Conference on Medical Image Computing and Assisted Intervention MICCAI BraTS, 2018, pp. 83-90.

15. A. Işın, C. Direkoğlu, C, and M. Sah. Review of MRI-based brain tumor image segmentation using deep learning methods. Procedia Computer Science, Vol. 102, pp. 317-324, 2016.

16. R. Saouli, M. Akil, and R. Kachouri. Fully automatic brain tumor segmentation using end-to-end incremental deep neural networks in MRI images. Computer methods and programs in biomedicine, Vol. 166, pp. 39-49, 2018.

17. D. Sridhar, I.V. Murali Krishna. Brain tumor classification using discrete cosine transform and probabilistic neural network, IEEE International Conference on Signal Processing Image Processing \& Pattern Recognition (ICSIPR), 2013, pp 92-96.

18. K.D. Kharat, P.P. Kulkarni, and M. Nagori. Brain tumor classification using neural network based methods. International Journal of Computer Science and Informatics, I (4), 2012.

19. S. Qamar, H. Jin, R. Zheng, and P. Ahmad. 3D Hyper-Dense Connected Convolutional Neural Network for Brain Tumor Segmentation. In 2018 14th International Conference on Semantics, Knowledge and Grids (SKG), 2018, pp. 123-130.

20. B. Cui, M. Xie, and C. Wang. A Deep Convolutional Neural Network Learning Transfer to SVM-Based Segmentation Method for Brain Tumor. In 2019 IEEE 11th International Conference on Advanced Infocomm Technology (ICAIT), 2019, pp. 1-5.

21. Z. Xiao, R. Huang, Y. Ding, T. Lan, R. Dong, Z. Qin, ... and $\mathrm{W}$. Wang. A deep learning-based segmentation method for brain tumor in MR images. In 2016 IEEE 6th International Conference on Computational Advances in Bio and Medical Sciences (ICCABS), 2016, pp. 1-6.

22. S. Pereira, A. Oliveira, V. Alves, and C.A. Silva. On hierarchical brain tumor segmentation in MRI using fully convolutional neural networks: a preliminary study. In 2017 IEEE 5th Portuguese meeting on bioengineering (ENBENG), 2017, pp. 1-4.

23. Z. Sobhaninia, S. Rezaei, N. Karimi, A. Emami, and S. Samavi. Brain Tumor Segmentation by Cascaded Deep Neural Networks Using Multiple Image Scales. arXiv preprint arXiv: 2002.01975, 2020.

24. L. Wu, Y. Xin, S. Li, T. Wang, P.-A. Heng and D. Ni, "Cascaded Fully Convolutional Networks for automatic prenatal ultrasound image segmentation," in IEEE 14th International Symposium on Biomedical Imaging (ISBI 2017), 2017.

25. A. Chaurasia, and E. Culurciello. LinkNet: Exploiting Encoder Representations for Efficient Semantic Segmentation, in IEEE Visual Communications and Image Processing (VCIP), 2017, pp. 1-4. 
Nurul Nadhirah Md Nasriy et al., International Journal of Advanced Trends in Computer Science and Engineering, 9(1.4), 2020,569 - 575

https://doi.org/10.1109/VCIP.2017.8305148

26. Y. Wang, C. Li, T. Zhu and C. Yu. A Deep Learning Algorithm for Fully Automatic Brain Tumor Segmentation. In 2019 International Joint Conference on Neural Networks (IJCNN), 2019, pp. 1-5. IEEE.

27. B. H. Menze, A. Jakab, S. Bauer, J. Kalpathy-Cramer, K. Farahani, J. Kirby, Y. Burren, N. Porz, J. Slotboom, and R. Wiest, "The multimodal brain tumor image segmentation benchmark (brats)," IEEE transactions on medical imaging, Vol. 35, no. 4, pp. 933-946, 2016.

28. Z. Akkus, A. Galimzianova, A. Hoogi, D. L. Rubin, and B. J. Erickson, "Deep learning for brain mri segmentation: state of the art and future directions," Journal of digital imaging, Vol. 30, no. 4, pp. 449-459, 2017. 